\title{
Controversies in migraine treatment: opioids should be avoided
}

\author{
G. Casucci $\cdot$ S. Cevoli
}

(C) Springer-Verlag Italia 2013

\begin{abstract}
The use of opioids for migraine is still controversial. Evidence-based guidelines do not recommend opioids as first-line treatment of migraine attacks, while clinical and epidemiological surveys demonstrate that the use of opioids is associated with more severe headacherelated disability, symptomology and comorbidities, and greater health-care resource utilization. There are concerns that opioids may be misused or abused, leading to opioid abuse or dependence and migraineurs are particularly prone and at risk for the development of chronic daily headache from opioids overuse. Since clinical and preclinical studies evidence a pathophysiological role of opioids in migraine progression, opioids should be avoided in migraine patients.
\end{abstract}

Keywords Opioid Medication overuse headache . Migraine $\cdot$ Hyperalgesia

\section{The role of opioids in clinical practice for migraine: the state-of-the-art}

Migraine is a common disabling neurologic disorder, characterized by recurrent headache attacks manifesting with unilateral location, pulsating quality, moderate or severe intensity, aggravation by routine physical activity, and association with nausea and/or photophobia and

G. Casucci $(\bowtie)$

Casa di Cura S. Francesco, Viale Europa 21,

82037 Telese Terme, Italy

e-mail: gerardocasucci@tin.it

S. Cevoli

IRCCS of Neurological Sciences of Bologna,

Bellaria Hospital, Via Altura 3, 40139 Bologna, Italy phonophobia [1]. The goals of acute migraine treatments are to treat attacks rapidly and consistently without recurrence, restore the patient's ability to function, minimize the use of back-up and rescue medications, optimize self-care and reduce subsequent use of resources, be cost-effective for overall management, and have minimal or no adverse events [2]. Acute treatments used for migraine include nonspecific agents such as aspirin, acetaminophen, non-steroidal anti-inflammatory drugs (NSAIDs), opioids, and barbiturates among others, as well as migraine-specific medications, including ergot alkaloids and triptans [2]. International guidelines recommend triptans and NSAIDs as first-line treatment of migraine attacks [2-4]. Use of opioids for treatment of migraine is controversial. Despite evidence-based guidelines do not recommend opioids as first-line treatment of migraine attacks [2-4], and no randomized controlled trial has shown any significant effect of opioids on migraine attacks when pain-free was the primary end-point of the study [5], their use in clinical practice [6-8] and, even more, in emergency departments (EDs) is very large, especially in USA and Canada $[9,10]$. Moreover, among studies about the efficacy of opioids as rescue medication for acute migraine in EDs recently revised, only one evidenced a superiority of meperidina $75 \mathrm{mg}$ i.m. versus ketorolac $30 \mathrm{mg}$ i.m., three reported superiority of competitors and eight reported similar results for opioids versus competitors such as DHE, metoclopramide, chlorpromazine, droperidol, and ketorolac [5]. In any case, opioids did not adequately restore the patient's ability to function. The negative impact of opioids on migrainous population is reported in several clinical and epidemiological studies. In fact, opioids use for migraine resulted associated with more severe headache-related disability, symptomology, and comorbidities (primarily, psychiatric, and cardiovascular), and greater health-care resource 
utilization. Repeat visitors to EDs were more likely to receive opioid medications, to use multiple doses of opioids, and to use opioids as initial treatment [6]. Recently, Tornabene et al. [11] showed that patients who received opioids for headache spent more time in ED than patients who did not. Moreover, those who received opioids as firstline treatment of migraine attacks in ED were significantly more likely to return to the same ED with a headache within 7 days of the original visit. Data from American Migraine Prevalence and Prevention study analyzing rates and reasons for discontinuation of triptans and opioids in episodic migraine from 2008 to 2009 showed that opioids use was associated with an increased risk of medication discontinuation compared to triptans. Reasons for medication discontinuation included return of migraine pain, concerns regarding drug interactions, and stomach upset, among others [6, 7]. The good management of acute migraine attacks is one of the most important components of the plan that prevents the transformation of episodic primary headache patterns into chronic headache disorders. Undoubtedly, there are concerns that opioids may be misused or abused, leading to opioids abuse or dependence $[12,13]$. Analgesic overuse has long been considered a risk factor for transformation of episodic migraine in chronic daily headache $(\mathrm{CDH})$ or medication overuse headache $(\mathrm{MOH})$ [14]. The $\mathrm{CDH}$ population includes individuals with chronic tension-type headache and chronic migraine $(\mathrm{CM})$, both of which may be associated with medication overuse $[15,16]$. $\mathrm{CDH}$ is a major clinical concern and a common health risk, with a prevalence of approximately $3-5 \%$ in the adult population worldwide. The answer to the question whether opioids overuse can lead to $\mathrm{MOH}$ or $\mathrm{CDH}$ was resolved in the ancillary study of Wilkinson et al. [17] who found that only patients with migraine who use daily opioids to control bowel motility after colectomy for ulcerative colitis, developed a transformed migraine. Patients without a history of migraine did not appear to be at risk for $\mathrm{CDH}$ even though they used daily opioids. These finding are indeed consistent with the concept that migraineurs are particularly prone and at risk for the development of $\mathrm{CDH}$ from opioids overuse. Other evidences supporting a role of opioids use as a risk factor for migraine chronification are provided by epidemiological studies. A prospective USA study of 8,219 migraineurs from the general population showed that 209 patients developed CM after 5 years of follow up [18, 19]. When compared with the use of acetaminophen, the use of opioids (and barbiturates) was found significantly associated with an increased risk of headache chronification, whereas the use of triptan or NSAIDs was not. The use of opioids at only 8 days/month was a risk factor for progression to $\mathrm{MOH}$. Data from the Frequent Headache Epidemiological Study confirm that opioids use was associated with $\mathrm{CDH}$
$(\mathrm{OR}=2.3,95 \% \mathrm{CI}=1.3-3.9)$ whereas aspirin and ibuprofen use were protective [20]. The last clinical evidence that opioids impact negatively on migraine attack is provided by a post hoc pooled analysis of rizatriptan clinical trials [21], demonstrating that recent prior opioids use was associated with lower triptans response.

\section{What is the action of opioids on the migraine brain?}

How opioids might cause MOH or transformed migraine and how they might interfere with effective acute migraine treatment is not completely known. Peripheral and central sensitization can explain the progression from episodic to chronic headache pain. In fact, cutaneous allodynia, a frequent event that develops during migraine attacks, reflects the decrease in nociceptive threshold, which is the key feature of sensitization [22]. There are many experimental evidences supporting a negative impact of opioids in migraine pathophysiology and progression. It is well known that long-term opioids use produces multiple adverse events among which opioid-induced hyperalgesia $(\mathrm{OIH})$, a state of nociceptive sensitization characterized by a paradoxical response whereby a subject receiving opioids become more sensitive to painful stimuli [23]. In case of $\mathrm{OIH}$, increasing opioids dosage would only worsen the pain that could be improved by reducing or eliminating the opioids itself. Interestingly, in headache patients OIH was reported even after brief exposure to opioids [24].

The neurobiological bases of $\mathrm{OIH}$ are not completely understood, but it is considered to result from neuroplastic changes in the peripheral and central nervous system that lead to sensitization of pronociceptive pathways. As recently reviewed [24], five mechanisms involving the central glutaminergic system, spinal dynorphins, descending facilitation, genetic liability, and decreased re-uptake of neurotransmitters along with enhanced nociceptive responsiveness, have been reported. Interestingly, all these mechanisms might be involved in the process of headache chronification. In particular, enhanced activation of the central glutaminergic system might be responsible of an increased cortical excitability [25]; in addiction, repeated morphine administration has also been shown to increase levels of pro-nociceptive peptides, as calcitonin generelated peptide (CGRP) and substance P, within the dorsal root ganglia in animal studies [26, 27]. Enhanced levels of CGRP and substance P might be present in dural afferents of trigeminal ganglia in migraine patients assuming opioids. These neuroadaptive changes may underlay hypersensitivity to normally non-noxious stimulation. Such pronociceptive neural adaptations might increase the response to triggers of migraine and enhance the frequency of attacks [28]. Other preclinical studies evidenced that 
sustained morphine exposure also induced a spinal dynorphin-dependent enhancement of excitatory transmitters release from primary afferent fibers. Moreover, in addition to the change in peripheral trigeminal afferents, animal studies demonstrate plasticity of central trigeminal neurons [29]. In morphine-treated rats, cutaneous receptive field sizes were larger, activation thresholds to electrical and mechanical stimulation of the dura were lower, and inhibition of heat-evoked activity by a remote noxious stimulus was abolished [30]. These results are consistent with an opioid-induced sensitization and a loss of Diffuse Noxious Inhibitory Controls (DNICs). The dysfunction of DNICs was also evidenced in patients with $\mathrm{MOH}$ [31]. In conclusion, opioids exposition in some predisposed migraine patients might produce neuroadaptive changes responsible for hyperexcitability of the trigeminal system and enhancement of descending facilitation throughout a loss of DNICs [28]. The development of drugs that do not interfere with such changes is of primary importance.

\section{Conclusion}

Opioids should not be used in migraine treatment [32] for many reasons. First of all because their use is not evidencebased: moderate or severe migraine attacks should be treated with specific anti-migraine medications according to the main guidelines, which consider opioids only as second or third-tier therapy for migraine, following both simple analgesics and migraine-specific medications [2-4]. This stratified-care approach provides use of triptans in moderate-to-severe migraine or in less severe migraine when non-opiate medications fail, and use of NSAIDs as first-line therapy for mild-to-moderate migraine [2]. Moreover, the use of opioids impacts negatively on migraine patients quality of life and it is responsible of a wastefulness of health-care resource. Last but not least, opioids are responsible for migraine progression. In Italy, the use of opioids is still limited respect to other European country due to a difficulty of prescription that was present since few years ago. A recent survey in an Italian ED evidences that tramadol is used in a small percentage of non-traumatic headache cases [33]. The Italian Law $38 / 2010$ assures the citizens right to have access to palliative care and to antalgic centers in case of either oncologic or non oncologic pain. The law gives directives about the simplification of procedures for the access to pain drugs such as opioids representing a risk for opioids overuse headache [34]. Drugs with psychotropic effects, such as opioids, are preferentially overused by chronic headache patients and are related to a dependence-related behavior, which in turn leads to a long-term opioids exposition [12]. In Italy, we have the opportunity to avoid the risk that the predictable diffusion of opioids, necessary for severe nononcologic pain but mainly ineffective in primary headaches, causes an increment of $\mathrm{MOH}$ incidence. For these reasons educational programs for migraine patients and the continuous professional development for physicians and all different healthcare professionals in headache area are warranted along with a continuous monitoring of opioids consumption for migraine in headache center and EDs.

Conflict of interest The author certifies that there is no actual or potential conflict of interest in relation to this article.

\section{References}

1. Headache Classification Committee of the International Headache Society (HIS) (2004) The international classification of headache disorders. 2nd edition. Cephalalgia 24(Suppl 1):1-160

2. Silberstein SD (2000) Practice parameter: evidence-based guidelines for migraine headache (an evidence-based review): report of the quality standards subcommittee of the american academy of neurology. Neurology 55:754-762

3. Scottish intercollegiate guidelines network guideline 107 (2008) Diagnosis and management of headache in adults. http://www. sign.ac.uk

4. NICE clinical guideline 150 (2012) Diagnosis and management of headaches in young people and adults. http://www.nice.org.uk

5. Kelley NE, Tepper DE (2012) Rescue therapy for acute migraine, part 3: opioids, NSAIDs, steroids, and post discharge medications. Headache 52:467-482

6. Buse DC, Pearlman SH, Reed ML, Serrano D, Ng-Mak DS, Lipton RB (2012) Opioid use and dependence among persons with migraine: results of the AMPP study. Headache 52:18-36

7. Bigal ME, Borucho S, Serrano D, Lipton RB (2009) The acute treatment of episodic and chronic migraine in the USA. Cephalalgia 29:891-897

8. Holland S, Fanning KM, Serrano D, Buse DC (2013) Rates and reasons for discontinuation of triptans and opioids in episodic migraine: results from the American migraine prevalence and prevention (AMPP) study. J Neurol Sci 326:7-10

9. Friedman BW, Grosberg BM (2009) Diagnosis and management of the primary headache disorders in the emergency department setting. Emerg Med Clin North Am 27:71-87

10. Colman I, Rothney A, Wright SC, Zilkalns B, Rowe BH (2004) Use of narcotic analgesics in the emergency department treatment of migraine headache. Neurology 62:1695-1700

11. Tornabene SV, Deutsch R, Davis DP, Chan TC, Vilke GM (2009) Evaluating the use and timing of opioids for the treatment of migraine headaches in the emergency department. J Emerg Med 36:333-337

12. Radat F, Lanteri-Minet M (2010) What is the role of dependencerelated behavior in medication-overuse headache? Headache 50:1597-1611

13. Praveen KT, Law F, O'Shea J, Melichar J (2011) Opioid dependence. Clin Evidence 09:1015

14. Evers S, Marziniak M (2010) Clinical features, pathophysiology, and treatment of medication-overuse headache. Lancet Neurol 9:391-401

15. Manzoni GC, Bonavita V, Bussone G, Cortelli P, Narbone MC, Cevoli S, D’Amico D, De Simone R, Torelli P, ANIRCEF (Associazione Neurologica Italiana Ricerca Cefalee) (2011) Chronic migraine classification: current knowledge and future perspectives. J Headache Pain 12:585-592 
16. Headache Classification Committee, Olesen J, Bousser MG, Diener HC, Dodick D, First M, Goadsby PJ, Göbel H, Lainez MJ, Lance JW, Lipton RB, Nappi G, Sakai F, Schoenen J, Silberstein SD, Steiner TJ (2006) New appendix criteria open for a broader concept of chronic migraine. Cephalalgia 26:742-746

17. Wilkinson SM, Becker WJ, Heine JA (2001) Opiate use to control bowel motility may induce chronic daily headache in patients with migraine. Headache 41:303-309

18. Bigal ME, Lipton RB (2008) Excessive acute migraine medication use and migraine progression. Neurology 71:1821-1828

19. Bigal ME, Serrano D, Buse D, Scher A, Stewart WF, Lipton RB (2008) Acute migraine medications and evolution from episodic to chronic migraine: a longitudinal population-based study. Headache 48:1157-1168

20. Scher AI, Lipton RB, Stewart WF, Bigal M (2010) Patterns of medication use by chronic and episodic headache sufferers in the general population: results from the frequent headache epidemiology study. Cephalalgia 30:321-328

21. Ho TW, Rodgers A, Bigal ME (2009) Impact of recent prior opioid use on rizatriptan efficacy: a post hoc pooled analysis. Headache 49:395-403

22. Bongsebandhu-phubhakdi S, Srikiatkhachorn A (2012) Pathophysiology of medication-overuse headache: implications from animal studies. Curr Pain Headache Rep 16:110-115

23. Lee M, Silverman SM, Hansen H, Patel VB, Manchikanti L (2011) A comprehensive review of opioid-induced hyperalgesia. Pain Physician 14:145-161

24. Saper JR, Lake AE 3rd (2008) Continuous opioid therapy (COT) is rarely advisable for refractory chronic daily headache: limited efficacy, risks and proposed guidelines. Headache 48:838-849

25. Coppola G, Schoenen J (2012) Cortical excitability in chronic migraine. Curr Pain Headache Rep 16:93-100
26. Ossipov MH, Lai J, Vanderah TW, Porreca F (2003) Induction of pain facilitation by sustained opioid exposure: relationship to opioid anti-nociceptive tolerance. Life Sci 73:783-800

27. Ossipov MH, Lai J, King T, Vanderah TW, Porreca F (2005) Underlying mechanisms of pronociceptive consequences of prolonged morphine exposure. Biopolymers 80:319-324

28. De Felice M, Ossipov MH, Porreca F (2011) Persistent medication-induced neural adaptations, descending facilitation and medication overuse headache. Curr Opin Neurol 24:193-196

29. Meng ID, Dodick D, Ossipov MH, Porreca F (2011) Pathophysiology of medication overuse headache: insights and hypotheses from preclinical studies. Cephalalgia 31:851-860

30. Okada-Ogawa A, Porreca F, Meng ID (2009) Sustained morphine-induced sensitization and loss of diffuse noxious inhibitory controls in dura-sensitive medullary dorsal horn neurons. J Neurosci 29:15828-15835

31. Perrotta A, Serrao M, Sandrini G, Burstein R, Sances G, Rossi P, Bartolo M, Pierelli F, Nappi G (2010) Sensitisation of spinal cord pain processing in medication overuse headache involves supraspinal pain control. Cephalalgia 30:272-284

32. Tepper SJ (2012) Opioids should not be used in migraine. Headache 52(Suppl 1):30-34

33. Torelli P, Campana V, Cervellin G, Manzoni GC (2010) Management of primary headaches in adult emergency departments: a literature review, the Parma ED experience and a therapy flow chart proposal. Neurol Sci 31:545-553

34. Cevoli S, Cortelli P (2011) Italian law "measures to guarantee the access to palliative and pain treatments": rebound on headaches management. Neurol Sci 32(Suppl 1):S77-S79 